\title{
AVALIAÇÃO SENSORIAL DOS FRUTOS DE CULTIVARES DE ABACAXI PARA CONSUMO IN NATURA ${ }^{1}$
}

\author{
SÁVIO DA SILVA BERILLI², SELMA BERGARA ALMEIDA³, \\ ALMY JUNIOR CORDEIRO DE CARVALHO ${ }^{3}$, SILVIO DE JESUS FREITAS ${ }^{4}$, \\ ANA PAULA CANDIDO GABRIEL BERILLI ${ }^{2}$, PAULO CESAR DOS SANTOS ${ }^{5}$
}

RESUMO - Os pomares de abacaxizeiro no Brasil sofrem constantemente com as perdas de plantas e frutos ocasionadas pela fusariose, pois as cultivares plantadas atualmente são suscetíveis a esta doença. Novas cultivares mais resistentes têm surgido, no entanto não há registros de que já tenham sido avaliadas sensorialmente por consumidores. O objetivo deste trabalho foi avaliar a aceitação sensorial de duas novas cultivares resistentes à fusariose, Vitória e EC-93, comparando-as com outras já estabelecidas no mercado consumidor de frutas in natura, Pérola e Gold. A aceitação dos frutos foi avaliada por 52 consumidores com relação a aroma, sabor, impressão global, textura, aparência da fatia, aparência do fruto inteiro, utilizando-se da escala hedônica estruturada de nove pontos, acidez e doçura ideais, com escala do ideal, e intenção de compra, com escala estruturada de 5 pontos. Sólidos solúveis totais, acidez titulável e ratio foram avaliados. A cultivar Gold obteve maiores médias de aceitação para a maior parte dos parâmetros avaliados, enquanto a cv EC93, as menores. Exceto no atributo aparência da fatia, a cv Vitória não diferiu das cultivares mais aceitas nos parâmetros avaliados. Os frutos diferiram significativamente quanto a SST, entre 12,0 e $16,0^{\circ}$ Brix, AT entre 0,52 e $0,81 \%$ de ácido cítrico e ratio entre 19,12 e 28,46. Dentre as cultivares resistentes à fusariose, os resultados sugerem baixo potencial de mercado para a cv EC-93 e bom para a cv Vitória.

Termos para indexação: Ananas comosus, cultivar Vitória, aceitação, fusariose, consumidor.

\section{SENSORY EVALUATION OF PINEAPPLE CULTIVARS FOR CONSUMPTION IN NATURA}

\begin{abstract}
The pineapple orchards in Brazil often presents losses of plants and fruits caused by fusariosis, because the current cultivars are susceptive to that disease. New resistant cultivars have been available. However, there are no records about their sensory evaluations by consumers. The objective of this work was to evaluate the sensory acceptability of two new cultivars resistant to Fusarium, Vitória and EC-93, by comparing them with other ones already established in the fresh fruits market, Pérola and Gold. The fruits were evaluated by 52 consumers using 9-points hedonic scale for aroma, flavor, texture, global impression, appearance of the slice, appearance of the fruit, using the right scale for ideal acidity and sweetness and using 5-points scale for purchase intention. It was also evaluated SS, ATTN and SS/AT. The cultivar Gold had the highest means of acceptance for most of the parameters evaluated, whereas the cv CS-93, the lowest. Except for the attribute appearance of slice, the Vitória cultivar did not differ from the most accepted cultivars in the evaluated parameters. The fruits differed significantly in the TSS, between 12.0 and $16.0^{\circ}$ Brix, TA between 0.52 and $0.81 \%$ citric acid and ratio between 19.12 and 28.46 . Among the cultivars resistant to Fusarium, the results suggest a low potential market for the EC-93 and a higher one for Vitória. Index terms: Ananas comosus, Vitória cultivars, acceptance, fusariosis, consumer.
\end{abstract}

\footnotetext{
${ }^{1}$ Resumo Sinfruit 181 - Simpósio Internacional de Fruticultura - Avanços na Fruticultura (17 a 21 Outubro)

${ }^{2}$ Professor/pesquisador do Instituto Federal do Espírito Santo - Câmpus Itapina

${ }^{3}$ Professor/pesquisador (a) da Universidade Estadual do Norte Fluminense

${ }^{4}$ Eng. Agr. Doutor em Produção Vegetal, Universidade Estadual do Norte Fluminense

${ }^{5}$ Eng. Agr. Mestrando em Produção Vegetal, Universidade Estadual do Norte Fluminense
} 


\section{INTRODUÇÃO}

O Brasil é grande consumidor e um dos maiores produtores mundiais de abacaxi. No entanto, o consumo de frutas frescas encontra-se abaixo das recomendações atuais da OMS, Organização Mundial de Saúde (OMS, 2004). As cultivares Pérola, Jupi e Smooth Cayenne são as mais plantadas no País (GONÇALVES, 2000).

O consumidor brasileiro de frutas aponta a aparência do fruto como a característica mais relevante no momento da compra, embora a renda e o preço também afetem o consumo (TEIXEIRA et al., 2006). No caso do abacaxi, a maior parte dos consumidores prioriza a coloração da fruta, seguida da firmeza da polpa, do tamanho e da aparência, tendo a acidez do fruto como a principal causa de descontentamento (MIGUEL et al., 2007).

Muitos programas de melhoramento genético vêm desenvolvendo novas cultivares, visando a superar dificuldades fitossanitárias da cultura do abacaxi, como a fusariose. Assim, algumas cultivares foram lançadas nos últimos anos, dentre as quais as cultivares: Imperial, em 2003, Vitória, em 2006, e IAC Fantástico, em 2010, todas com promissoras características para consumo in natura e resistentes à fusariose (MAPA, 2004; INCAPER, 2006; IAC, 2010).

As cultivares de abacaxi existentes atualmente no mercado mundial e brasileiro, como Smooth Cayenne, Pérola, Havaí e Gold, já estão elucidadas quanto aos aspectos de qualidade dos frutos e algumas sensorialmente, de forma que a cultivar Pérola é a mais atrativa ao consumidor brasileiro, com características preferidas de doçura, maciez, odor, baixa acidez e sabor agradável em comparação a outras cultivares (CHAN et al., 2002; REINHARDT et al., 2004; INCAPER, 2006; BENGOZI et al., 2007; MIGUEL at al., 2007).

Alguns trabalhos vêm comparando o desempenho dos atributos qualitativos físicos, químicos e sensoriais entre diferentes cultivares, de modo que, na maior parte das vezes, a cultivar Pérola é a que apresenta melhor desempenho no mercado doméstico (CUNHA et al., 2007; BRITO et al., 2008).

A cultivar Vitória apresenta características similares à cultivar Pérola, incluindo a coloração da polpa. A cv EC-93 é um híbrido aberto resultante do cruzamento do Manzana, cv originária da Colômbia, com o Perolera, apresentando características de espinhos nas folhas, muito doce, com aderência dos filhotes na base do fruto e resistente à fusariose. Entretanto, não há relatos na literatura científica envolvendo avaliações sensoriais dessas cultivares, sobretudo do ponto de vista do consumidor. O objetivo deste trabalho foi avaliar a aceitação sensorial de duas novas cultivares resistentes à fusariose, Vitória e EC-93, comparando-as com outras já estabelecidas no mercado consumidor de frutas in natura, Pérola e Gold.

\section{MATERIAL E MÉTODOS}

\section{Amostras}

As quatro cultivares de abacaxi foram: Gold e EC-93, de polpa amarela, e Pérola e Vitória, de polpa branca, sendo as cultivares Vitória e EC-93 resistentes à fusariose.

Os frutos foram cultivados na estação experimental de Bananal do Norte, do Incaper (Instituto Capixaba de Pesquisa, Assistência Técnica e Extensão Rural), município de Cachoeiro de Itapemirim (ES). As coordenadas são: $20^{\circ} 75^{\prime}$ de latitude sul, e $41^{\circ} 29^{\prime}$ de longitude oeste, altitude de $146 \mathrm{~m}$. O solo é um Latossolo Vermelho-Amarelo de textura média, com irrigação suplementar, em que todas as cultivares foram plantadas de mudas tipo filhote no mesmo período (junho de 2008) e mesmo espaçamento ( 90 x $30 \mathrm{~cm}$ ), tendo recebido os mesmos tratos culturais, com indução natural nos meses de julho a agosto de 2009. Os frutos foram colhidos ao acaso, em um mesmo momento, com estádio de maturação superior a $90 \%$ da casca amarela, sendo todas as avaliações efetuadas seis dias após a colheita.

\section{Aceitação Sensorial}

Cinquenta e dois indivíduos que gostavam de abacaxi in natura em grau igual ou superior a moderadamente, entre estudantes, funcionários e professores da UENF, com idade a partir de 19 anos, sendo $51 \%$ do sexo feminino e $49 \%$ do sexo masculino, foram recrutados para participar do teste, a partir das respostas a um questionário.

Foram utilizadas as seguintes escalas nas avaliações: escala hedônica estruturada mista de 9 pontos (PERYAM; GIRARDOT, 1952), para aceitação quanto ao aroma, sabor, textura, impressão global, aparência da fatia e aparência do fruto inteiro com casca e coroa; escala do ideal estruturada verbal de nove pontos (MEILGAARD et al., 2006), para intensidades dos gostos doce e ácid, e escala estruturada mista de 5 pontos (MEILGAARD et al., 2006), para intenção de compra.

O teste de aceitação foi realizado em nível laboratorial, em cabines individuais, sob iluminação vermelha, nas avaliações da aceitação do aroma, sabor, textura e impressão global, e sob luz branca fluorescente, nas avaliações da aceitação da aparên- 
cia do fruto inteiro, aparência da fatia e intenção de compra.

As amostras foram preparadas da seguinte forma: após descascar o fruto e remover completamente as olhaduras, eliminaram-se $3 \mathrm{~cm}$ das extremidades superior e inferior da polpa. Em seguida, a parte intermediária foi fatiada em rodelas de $1,5 \mathrm{~cm}$ de espessura (aproximadamente $65 \mathrm{~g}$ ), as quais foram divididas radialmente em 8 partes, sendo servidos 3 pedaços para cada consumidor, nas avaliações sob luz vermelha. Para avaliação da aparência da fatia, foi utilizada uma rodela da polpa $(1,5 \mathrm{~cm}$ de espessura), da parte mediana do fruto. Todas as amostras foram servidas em pratos descartáveis brancos, codificados com números aleatórios de três dígitos, acompanhadas de água e biscoito de água e sal.

A ordem de apresentação das amostras seguiu delineamento descrito por Macfie et al.(1989), para minimização do efeito "first-order carry-over", que é o efeito da avaliação de uma amostra sobre a avaliação da amostra subsequente.

\section{Análises Físico-químicas}

Os teores de sólidos solúveis totais (SST), acidez titulável (AT) e o ratio, que é a relação SST/ AT, foram determinados nas polpas dos frutos, seguindo metodologias descritas pela AOAC (1997).

\section{Análises Estatísticas}

Os dados sensoriais e os resultados das análises físico-químicas foram submetidos à análise de variância $(p \leq 0,05)$ e teste de Tukey $(p \leq 0,05)$, utilizando o programa estatístico SAS - Statistical Analysis System, versão 9.3. Todos os dados sensoriais foram também avaliados através da distribuição de frequência das respostas dos consumidores.

\section{RESULTADOS E DISCUSSÃO}

Houve diferença significativa ( $\mathrm{p} \leq 0,05)$ entre as cultivares, em todos os parâmetros avaliados (Tabela 1).

De forma geral, a cultivar EC-93 foi a menos aceita em todos os parâmetros avaliados, enquanto as cultivares Gold e Vitória foram as mais bem aceitas pelos consumidores, com relação aos atributos aroma, sabor, textura e impressão global, obtendo também atitude de compra positiva (Tabela 1). Por outro lado, na avaliação da aceitação da aparência da fatia, a cv Pérola revelou-se superior às demais, e na aparência do fruto, esta e a cv Vitória. Mesmo essas duas cultivares diferindo com relação à aceitação da aparência da fatia, esses resultados confirmam a preferência do consumidor brasileiro por abacaxi de polpa branca, conforme comentado por Carvalho et al. (1998).

Quando avaliadas as distribuições de frequência das respostas dos consumidores com relação aos parâmetros avaliados, a cv EC-93 obteve maiores proporções de respostas na região de rejeição da escala hedônica (entre desgostei extremamente/ detestei e desgostei ligeiramente), como de atitude negativa de compra (entre certamente não compraria e certamente compraria). Por outro lado, as outras cultivares apresentaram predominância de respostas em categorias igual ou superiores a gostei moderadamente em todos os atributos hedônicos avaliados (Figuras 1 e 2).

Assim, as cultivares de polpa branca, Pérola e Vitória, apresentaram-se similares com relação à intenção de compra e à aceitação do aroma, sabor, textura, impressão global e aparência do fruto (Tabela 1). Brito et al. (2008) relataram a similaridade da aceitação da aparência, aroma, sabor, textura e impressão global da polpa da cultivar Pérola pelo consumidor em relação à de outras cultivares, IAC-Gomo-de-mel e Smooth Cayenne.

$\mathrm{Na}$ Tabela 1, destacam-se os parâmetros textura e intenção de compra, cujas avaliações resultaram em apenas dois segmentos de médias. O primeiro, formado pelas cultivares Vitória, Pérola e Gold, com maiores médias, e o segundo, pela cv EC-93. De forma similar à avaliação dos atributos hedônicos, observou-se predominância das respostas quanto à intenção de compra das cultivares Vitória, Pérola e Gold na região positiva de compra (77\%, $71 \%$ e $63 \%$, respectivamente - Figura 3 ). Lembrando que as avaliações desses dois parâmetros foram realizadas separadamente e, na forma cega, uma não teve efeito sobre a outra. Assim, diante desses resultados, seria interessante caracterizar, por meio de medidas instrumentais e/ou sensoriais, a textura da polpa dessas cultivares para se correlacionar com a aceitação atribuída pelos consumidores.

Em contrapartida, certamente foi a aceitação da aparência dos frutos que influenciou na intenção de compra dos consumidores, uma vez que as duas avaliações ocorreram conjuntamente. Entretanto, na avaliação da aceitação da aparência, houve maior segmentação entre as médias (Tabela 1). Contudo, as diferenças na avaliação da aceitação da aparência dos frutos das cultivares Vitória, Pérola e Gold não foram suficientes para segmentá-las na avaliação da intenção de compra. Vale ressaltar que as avaliações da aparência dos frutos ocorreram de forma isolada das demais avaliações, de modo que os consumidores não associavam a coloração ou outras características da polpa com a aparência externa do fruto. 
Os resultados discutidos anteriormente sugerem favorável comercialização da cv Vitória e desfavorável da cv EC-93, visto que a aparência do fruto é uma das principais características consideradas pelos consumidores no momento da compra (CARVALHO et al., 1998; MIGUEL at al., 2008). De acordo com Miguel at. al. (2008), outras características também são relevantes ao consumidor brasileiro no ato da compra, como firmeza da polpa (externamente), tamanho do fruto, estado de conservação e defeitos dos frutos.

Os maiores teores de SST foram observados nas cultivares Vitória e Gold, que obtiveram maiores médias nos atributos hedônicos aroma, sabor e impressão global (Tabelas 1 e 2).

Apesar de maiores teores de SST e de AT nos alimentos resultarem em maiores doçura e acidez, respectivamente, a percepção sensorial dessas características em frutas e sucos não devem ser consideradas isoladamente, visto que uma interfere na percepção da outra, conforme já relatado por outros autores (MANGIAVACCHI; ALMEIDA, 2010; BERGARA-ALMEIDA, 2002; CHITARRA;CHITARRA, 1990). Portanto, a relação desses dois parâmetros (SST/AT), o ratio, pode ser a expressão dos teores de SST e AT no abacaxi mais indicada para correlacionar esses parâmetros com a percepção da doçura e acidez sensorial. Entretanto, para os atributos hedônicos sabor e impressão global, o teor de SST foi preponderante.

Aproximadamente $50 \%$ dos consumidores consideraram ideal a doçura da polpa do fruto da cv Gold, e $40 \%$ a da cv Vitória, enquanto para a acidez, 42 e $22 \%$, respectivamente (Figura 4). Possivelmente, o elevado ratio da polpa dos frutos da cv Gold contribuiu para suas maiores médias de aceitação sensorial quanto ao sabor, sugerindo que os valores do ratio do fruto devem estar próximos ao observado para esta cultivar (Tabelas 1 e 2; Figura 4).

Brito et al. (2008) verificaram que a cultivar IAC-Gomo-de-mel apresentou teor de SST significativamente superior ao das cultivares Pérola e Smooth Cayenne, não observando diferença significativa $(\mathrm{p} \leq$ $0,05)$ quanto à aceitação sensorial do sabor da polpa e da impressão global, tal como verificado neste trabalho entre as cultivares Vitória e Gold (Tabela 1 e Figura 4). Além disso, é possível considerar que outras características químicas, provavelmente distintas entre os frutos das cultivares de abacaxi, como a composição em compostos voláteis responsáveis por aroma e sabor ou a presença de substâncias que resultam em gosto amargo ou em outras características sensoriais, podem ter influência na resposta de aceitação do aroma, sabor e impressão global dos consumidores. Portanto, é necessária a realização de pesquisas complementares que se concentrem nessas investigações.

TABELA 1 - Médias $^{1}$ de aceitação e intenção de compra atribuídas pelos consumidores ( $\mathrm{n}=52$ ) às quatro cultivares de abacaxi para consumo in natura.

\begin{tabular}{cccccccc}
\hline Cultivar & Aroma $^{2}$ & Sabor $^{2}$ & Textura $^{2}$ & $\begin{array}{c}\text { Impressão } \\
\text { Global }^{2}\end{array}$ & $\begin{array}{c}\text { Aparência } \\
\text { da fatia }^{2}\end{array}$ & $\begin{array}{c}\text { Aparência } \\
\text { do fruto }^{2}\end{array}$ & $\begin{array}{c}\text { Intenção de } \\
\text { compra }^{3}\end{array}$ \\
\hline Vitória & $6,6 \mathrm{ab}$ & $6,9 \mathrm{ab}$ & $7,2 \mathrm{a}$ & $7,1 \mathrm{ab}$ & $6,0 \mathrm{~b}$ & $7,2 \mathrm{a}$ & $4,3 \mathrm{a}$ \\
Pérola & $5,9 \mathrm{~b}$ & $6,5 \mathrm{~b}$ & $7,1 \mathrm{a}$ & $6,7 \mathrm{bc}$ & $7,3 \mathrm{a}$ & $6,7 \mathrm{ab}$ & $4,1 \mathrm{a}$ \\
Gold & $7,2 \mathrm{a}$ & $7,7 \mathrm{a}$ & $7,4 \mathrm{a}$ & $7,4 \mathrm{a}$ & $5,1 \mathrm{~b}$ & $6,3 \mathrm{~b}$ & $3,8 \mathrm{a}$ \\
EC-93 & $6,1 \mathrm{~b}$ & $5,4 \mathrm{c}$ & $6,2 \mathrm{~b}$ & $6,0 \mathrm{c}$ & $5,6 \mathrm{~b}$ & $4,7 \mathrm{c}$ & $2,6 \mathrm{~b}$ \\
\hline
\end{tabular}

${ }^{1}$ médias iguais numa mesma coluna não diferem significativamente $(\mathrm{p} \leq 0,5)$, pelo teste de Tukey; ${ }^{2} 1$ : desgostei extremamente / detestei; 5: nem gostei / nem desgostei: 9: gostei extremamente / adorei; ${ }^{3} 1$ : certamente não compraria; 5: certamente compraria.

TABELA 2 - Valores médios* de sólidos solúveis (SST), acidez titulável (AT) e razão SST/AT (ratio) de quatro cultivares de abacaxi in natura.

\begin{tabular}{cccc}
\hline Cultivar & SST $\left({ }^{\circ}\right.$ Brix $)$ & $\begin{array}{c}\text { AT } \\
\text { (\% ác. cítrico) }\end{array}$ & SST/AT \\
\hline Vitória & $16,00 \mathrm{a}$ & $0,81 \mathrm{a}$ & 19,80 \\
Pérola & $13,07 \mathrm{c}$ & $0,59 \mathrm{bc}$ & 22,17 \\
Gold & $14,85 \mathrm{~b}$ & $0,52 \mathrm{c}$ & 28,46 \\
EC-93 & $12,02 \mathrm{~d}$ & $0,63 \mathrm{~b}$ & 19,12 \\
\hline CV $(\%)$ & 3,96 & 10,48 & \\
\hline
\end{tabular}

Médias iguais numa mesma coluna não diferem significativamente $(\mathrm{p} \leq 0,05)$, pelo teste de Tukey. 

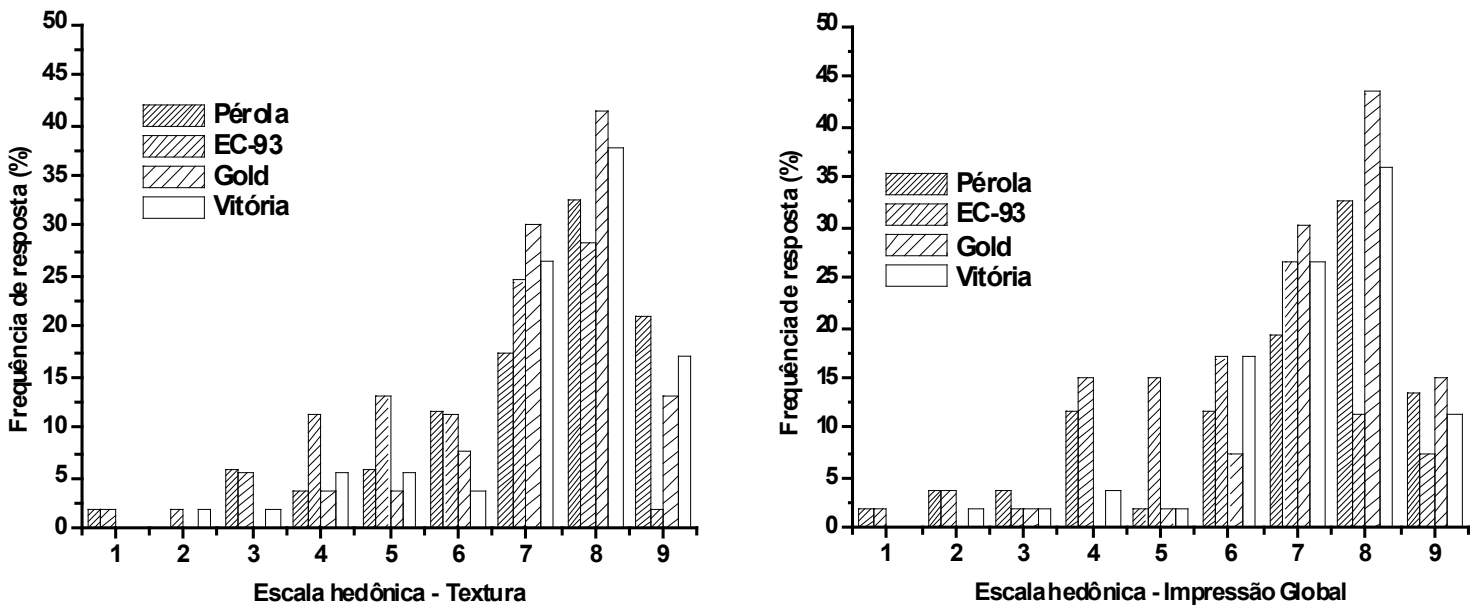

FIGURA 1 - Distribuição de frequência das respostas dos consumidores (\%) para a aceitação de textura e impressão global dos frutos de quatro cultivares de abacaxi (1: desgostei extremamente/detestei; 5: nem gostei/nem desgostei: 9: gostei extremamente/ adorei).
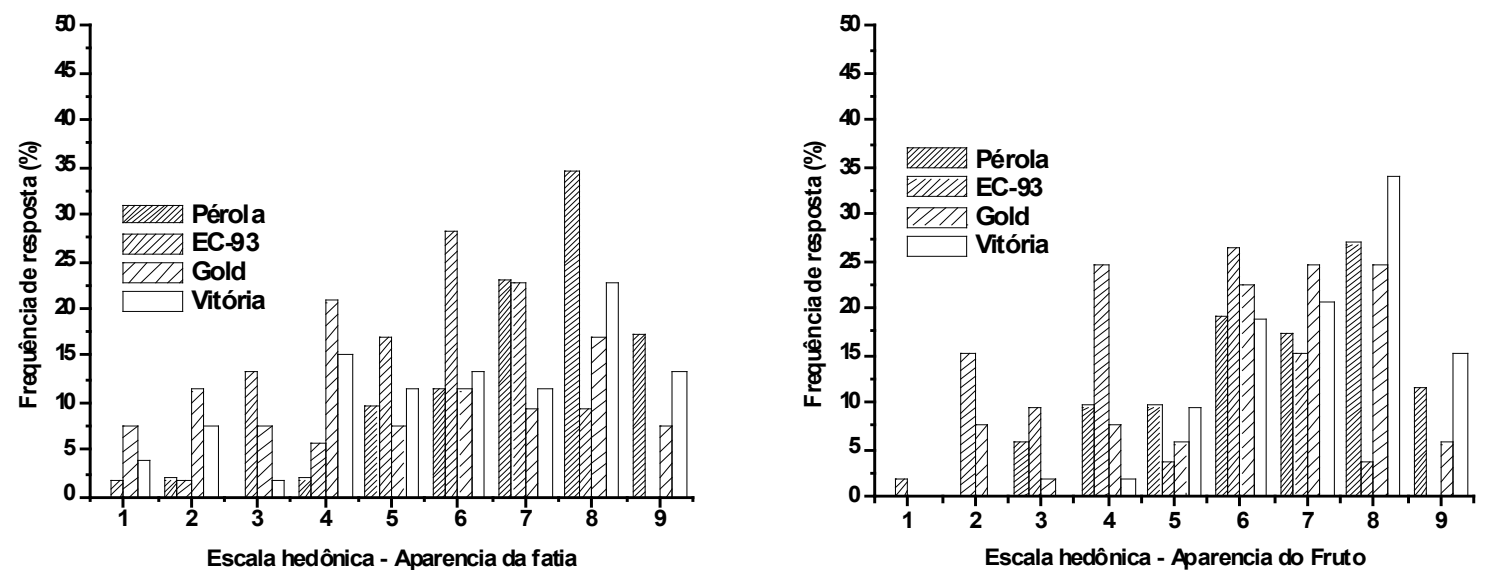

FIGURA 2 - Distribuição de frequência das respostas dos consumidores (\%) para a aceitação da aparência da fatia e dos frutos de quatro cultivares de abacaxi (1: desgostei extremamente/detestei; 5: nem gostei/nem desgostei: 9: gostei extremamente/ adorei).

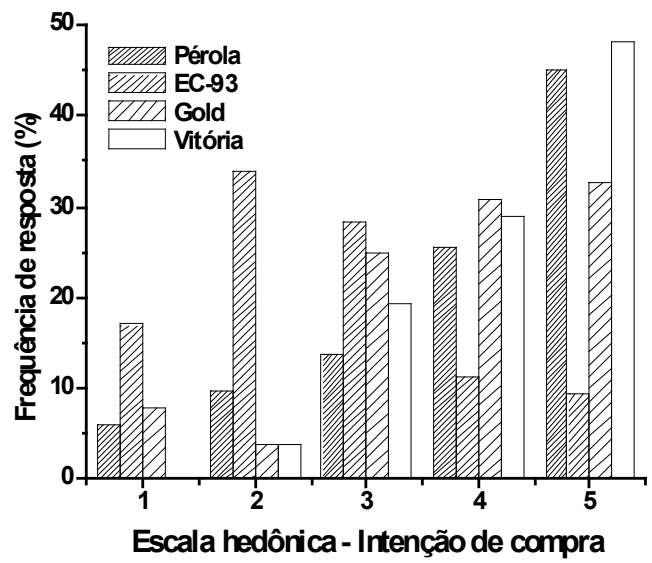

FIGURA 3 - Distribuição de frequência das respostas dos consumidores (\%) com relação à intenção de compra dos frutos das quatro cultivares de abacaxi (1: certamente não compraria; 5 : certamente compraria). 

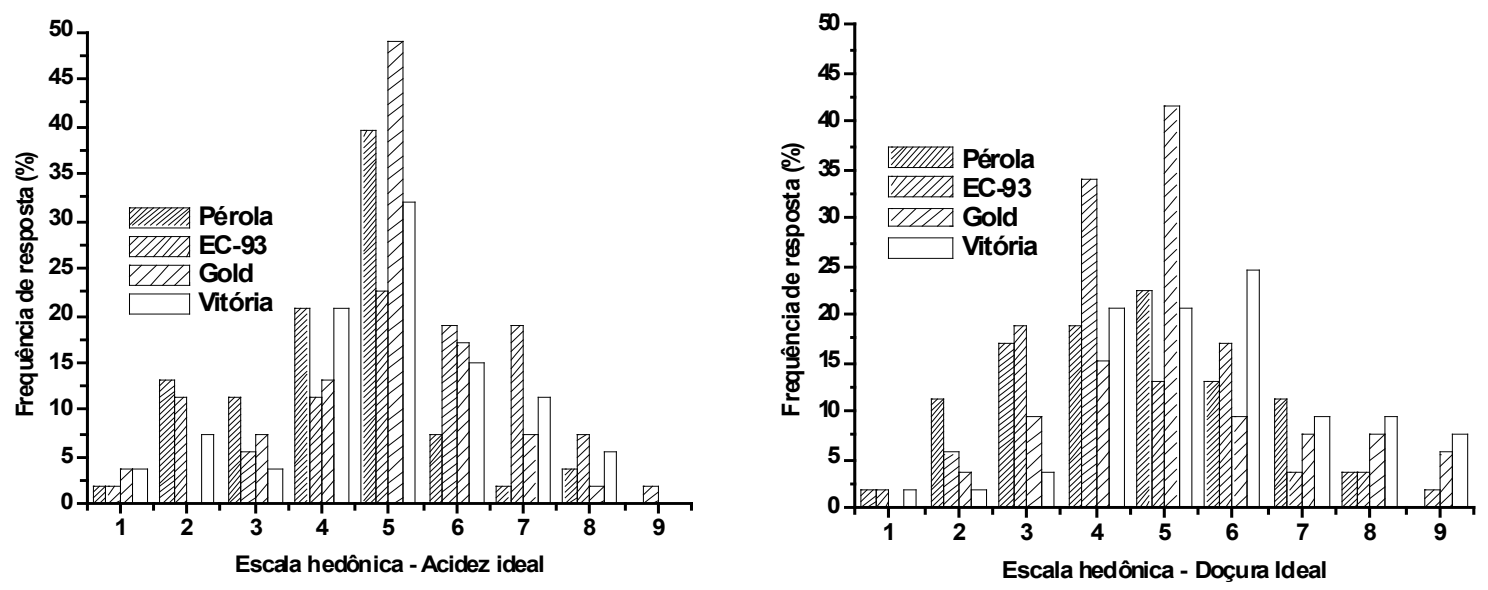

FIGURA 4 - Distribuição de frequência das respostas dos consumidores (\% de pessoas) em função da acidez e doçura ideal dos frutos de quatro cultivares de abacaxi $(1$ = extremamente mais ácida/ doce que o ideal; 5 = ideal; 9 = extremamente mais ácida/doce que o ideal).

\section{CONCLUSÃO}

Os resultados deste trabalho permitem inferir que a cultivar Vitória, resistente à fusariose, possui bom potencial de comercialização, visto que apresentou intenção de compra e aceitação quanto à aparência do fruto, aroma, sabor, textura e impressão global similar ou superior às atribuídas às cultivares Pérola e Gold, já estabelecidas no mercado consumidor. No entanto, ficou evidente o baixo potencial da cultivar EC-93, resistente à fusariose, ao mercado consumidor, uma vez que apresentou resultados inferiores em relação às outras cultivares em praticamente todos os parâmetros sensoriais avaliados neste trabalho. Ainda, com os resultados observados neste trabalho, confirma-se a preferência do consumidor brasileiro por abacaxis de polpa branca, como as cultivares Pérola e Vitória.

\section{REFERÊNCIAS}

AOAC. Official methods of analysis of the Association of Official Analytical Chemists. $16^{\text {th }}$ ed. Washington, 1997. p. 2: 37-10, 42-42, 44-43, 45-16.

CUNHA, G. A. P.; CABRAL, J. R. S.; MATOS, A. P.; CALDAS, R. C. Avaliação de genótipos de abacaxi resistentes à fusariose em coração de Maria, Bahia. Magistra, Cruz Almas, v. 19, n. 3, p. 219-223, 2007.

BENGOZI, F. J.; SAMPAIO, A. C.; SPOTO, M. H. F.; MISCHAN, M. M.; PALLAMIN, M. L. Qualidades físicas e químicas do abacaxi comercializado na Ceagesp - Jaboticabal. Revista Brasileira de Fruticultura, Jaboticabal, v. 29, n. 3, p. 540-545, 2007.
BERGARA-ALMEIDA, S.; DA SILVA, M. A. A. P. Hedonic scale with reference: performance in obtaining predictive models. Food Quality and Preference, Barking, n. 13, v. 1, p. 57-64, 2002.

BRITO, C. A. K.; SIQUEIRA, P. B.; PIO, T. F.; BOLINI, H. M. A.; SATO, H. H. Caracterização físico-química, enzimática e aceitação sensorial de três cultivares de abacaxi. Ponta Grossa. Revista Brasileira de Tecnologia Agroindustrial, Ponta Grossa, v. 2, n. 2, p. 1-14, 2008.

CARVALHO, V. D.; ABREU, C. M. P.; GONÇALVES, N. B. Qualidade e industrialização do abacaxi. Belo Horizonte. Informe Agropecuário, Belo Horizonte, v.195, p.67-69, 1998.

CHAN, Y. K.; COOPENS, E. G.; SANEWSKI, G. $M$. Breeding and variety improvement. Honolulu: University of Hawaii at Manoa, 2002. p. 33-55.

CHITARRA, M. I. F.; CHITARRA, A. B. Pós-colheita de frutos e hortaliças: fisiologia e manuseio. Lavras: ESAL-FAEPE, 1990. 320 p.

GONÇALVES, N. B. E.; CARVALHO, V. D. Características da fruta. In: GONÇALVES, B. N. Abacaxi: pós-colheita. Brasília: Embrapa, 2000. 45p.

IAC- Instituto Agronômico de Campinas. São Paulo lança cultivar de abacaxi IAC Fantástico para substituir cultivares em uso no Brasil. 2010. Disponível em: <http://www.iac.sp.gov.br/conteudo_noticias_pop.asp?id=606>. Acesso em: $02 \mathrm{fev} .2010$. 
INCAPER. Nova cultivar de abacaxi resistente à fusariose. Vitória, 2006. (Documento, 148)

MACFIE, H. J.; BRATCHELL, N.; GREENHOFF, K.; VALLIS, L. Designs to balance the effect of order of presentation and first-order carry-over effects in hall tests. Journal of Sensory Studies, Westport, v. 4, n. 2, p. 129-148, 1989.

MANGIAVACCHI, P. M.; ALMEIDA, S. B. Otimização da aceitação sensorial de suco de maracujá-amarelo em função da diluição da polpa e dos teores de sólidos solúveis e acidez In: ENCONTRO DE INICIAÇÃO CIENTÍFICA DA UENF, 15., 2010. Campos dos Goytacazes. Anais...

MAPA. Novo híbrido resistente à fusariose é lançado na Paraíba. Brasília: Embrapa, 2004. Disponível em: <http://www.embrapa.gov.br/imprensa/ noticias/2003/maio/bn.2004-11-25.1029961427/>. Acesso em: 04 fev. 2010.

MEILGAARD, M.; CIVILLE, G.V.; CARR, B.T. Sensory evaluation techniques. 4.ed. Boca Raton: CRC Press, 2006. 448 p.

MIGUEL, A. C. A.; SPOTO, M. H. F.; ABRAHÃO, C.; SILVA, P. P. M. Aplicação do método QFD na avaliação do perfil do consumidor de abacaxi pérola. Ciência Agrotécnica, Lavras., v. 31, n. 2, p. 563 569, 2007

OMS - World Health Organization. Global strategy on diet, physical activity and health. In: WORLD HEALTH ASSEMBLY, 57., 2004. Geneva. Proceedings...

PERYAM, D. R.; GIRARDOT, N. Advanced tastetest method. Food Engineering, New York, v. 24, n. 7, p. 58-61, 1952.

REINHARDT, D. H.; MEDINA, V. M.; CALDAS, R. C.; CUNHA, G. A. P.; ESTEVAM, R. F. H. Gradientes de qualidade em abacaxi Pérola em função do tamanho e do estádio de maturação do fruto. Jaboticabal. Revista Brasileira de Fruticultura, Jaboticabal, v. 26, n.3, p. 544-546, 2004.

TEIXEIRA, L. J. Q.; PEREIRA, J. M. A, T. K.; SILVA, N. M.; REIS, F. P. Hábitos de consumo de frutas entre estudantes da Universidade Federal de Viçosa. Ceres, Viçosa, MG, v.53, n. 307, p. 366-373, 2006. 